\title{
P84 Prediction of Cardiovascular Events and Mortality by Pulse Wave Velocity: a Comparison of Invasive Measurements and Noninvasive Estimation Methods
}

Bernhard Hametner ${ }^{1, *}$, Siegfried Wassertheurer ${ }^{1}$, Christopher Clemens Mayer ${ }^{1}$, Kathrin Danninger ${ }^{2}$, Ronald Binder ${ }^{2}$, Thomas Weber ${ }^{2}$

${ }^{1}$ Center for Health \& Bioresources, AIT Austrian Institute of Technology, Vienna, Austria

${ }^{2}$ Cardiology Department, Klinikum Wels-Grieskirchen, Wels, Austria

\begin{abstract}
Introduction: Arterial properties are linked to the presence and severity of coronary artery disease (CAD). Coronary blood flow is strongly and inversely related to arterial stiffness. Thus, the aim of this prospective study was to evaluate the prognostic value of PWV in high-risk patients with suspected CAD and to compare three different methods for assessing PWV.

Methods: In 1040 patients, invasive PWV (iPWV) was measured during catheter pullback. Additionally, PWV was estimated with a model incorporating age and the central pulse waveform obtained from non-invasive measurements (ePWV). As a third method, PWV was calculated with a formula solely based on age and blood pressure (fPWV). Survival analysis was done for continuous PWV as well as using cut-off values.

Results: After a median follow up duration of 1565 days, $24 \%$ of the patients reached the combined endpoint (cardiovascular events and mortality). Cox proportional hazard ratios per m/s were 1.13 for iPWV, 1.17 for ePWV and 1.13 for fPWV $(p<0.0001$ for all three methods). Significant differences between patient groups according to a cut-off value of $10 \mathrm{~m} / \mathrm{s}$ were found as well for all three methods. However, multivariable Cox models revealed a different performance of the three methods, indicating weaknesses of fPWV to mimic iPWV.

Conclusion: All three methods for the determination of PWV predicted cardiovascular events and mortality in patients with suspected coronary artery disease. This indicates that iPWV as well as both non-invasive estimation methods are suitable for the assessment of arterial stiffness, bearing in mind their individual characteristics.
\end{abstract}

(c) 2019 Association for Research into Arterial Structure and Physiology. Publishing services by Atlantis Press International B.V. This is an open access article distributed under the CC BY-NC 4.0 license (http://creativecommons.org/licenses/by-nc/4.0/) 